\title{
Trastornos mentales y su relación con la dinámica familiar en el departamento de Santa Bárbara
}

Nancy Martínez Cubas ${ }^{1}$

German Moncada ${ }^{2}$

\section{RESUMEN}

Este artículo tiene el objetivo de analizar la prevalencia de los trastornos mentales y su relación con la dinámica familiar en la población adulta del departamento de Santa Bárbara. Los métodos en la investigación se basa en un diseño descriptivo, cuantitativo, de corte trasversal con un muestreo polietápico con criterios de selección en donde fueron encuestadas 300 viviendas, utilizando como instrumentos el Cuestionario de Síntomas para adultos (SRQ) propuesto por la OMS para la detección de sintomatología probables trastornos mentales y el Cuestionario de funcionamiento familiar (FFSL) de Ortega y Colaboradores. Se trabajó con el programa SPSS para obtener los resultados analíticos de la investigación. Los trastornos mentales de mayor prevalencia son la depresión, seguido de estrés postraumático, ansiedad, dependencia a las drogas, en menor porcentaje se encuentra la dependencia al alcohol y epilepsia. La mayor frecuencia de los probables trastornos mentales es en el género femenino, así como las personas mayores de 51 años, de la misma forma los solteros (as). En relación a las familias de Santa Bárbara que presentan dinámica familiar funcional es decir que a mayor funcionamiento familiar menor presencia de trastornos mentales. En conclusión la prevalencia de probables trastornos mentales en el departamento de Santa Bárbara es mayor a lo reportado por la OMS (2002) por lo que representa un reto para el sistema de salud hondureño y demuestra la necesidad de enfocar la atención y recursos hacia la prevención, promoción y tratamiento oportuno de los trastornos mentales.

Palabras clave: trastornos mentales, prevalencia, dinámica familiar, funcionalidad familiar.

\footnotetext{
${ }^{1}$ Beneficiaria de una beca de estudiante de posgrado de la DICYP. Estudiantes de la Maestría en Psicología Clínica, Escuela de Ciencias Psicológicas, Facultad de Ciencias Sociales, Universidad Nacional Autónoma de Honduras: nancywais5@hotmail.com

${ }^{2}$ Asesor, Coordinador del área de investigación en la maestría de Psicología clínica, profesor investigador de Escuela de Ciencias Psicológicas de la Universidad Nacional Autónoma de Honduras: germanmoncada@yahoo.es.
} 


\section{ABSTRACT}

This article aims to analyze the prevalence of mental disorders and their relationship with family dynamics in the adult population of the department of Santa Barbara. The research methods are based on a descriptive, quantitative, cross-sectional design with a polyvalent sampling with selection criteria in which 300 houses were surveyed, using as instruments the WHO Symptom Questionnaire for Adults (SRQ), Detection of probable mental disorders symptomatology and the Family Functioning Questionnaire (FFSL) of Ortega and Co. We worked with the SPSS program to obtain the analytical results of the research. The most prevalent mental disorders were depression, followed by post-traumatic stress, anxiety, drug dependence. The less prevalent mentar disorders were alcohol dependence and epilepsy. The highest frequency of probable mental disorders is in the female gender, people over 51 years of age, and people who remain single. In relation to the families of Santa Bárbara that present functional family dynamics, that is to say, that the greater the family functioning, the less presence of mental disorders. In conclusion, the prevalence of probable mental disorders in the department of Santa Barbara is higher than that reported by the WHO (2002), which represents a challenge for the Honduran health system and demonstrates the need to focus the care and resources towards the prevention, promotion and treatment of mental disorders.

Key words: mental disorders, prevalence, family dynamics, family functionality 


\section{INTRODUCCIÓN}

El propósito de este artículo es describir la prevalencia de los trastornos mentales y su relación con la dinámica familiar, presentando datos epidemiológicos significativos sobre la situación del departamento de Santa Bárbara información que puede servir para la reorganización y elaboración de nuevas propuestas en salud mental con la intención de mejorar las condiciones de salud y desarrollo psicológico de este departamento. Igualmente la divulgación de estos resultados será una contribución para la formación del personal encargado de las intervenciones preventivas y para la solución de los problemas encontrados.

La relevancia social del estudio radica en el desarrollo de nuevas estrategias de intervención psicosocial que garanticen una atención accesible, descentralizada, participativa, continuada, y preventiva a favor de las comunidades detectadas con sintomatología de probables trastornos mentales o problemas relacionados con la dinámica familiar. De igual forma, dicha información permitirá adjudicar recursos en conformidad con las necesidades detectadas y abogar por los derechos de dicha población objeto de estudio.

La práctica de este estudio contribuirá a la mejora de la atención primaria en salud mental en las comunidades de Santa Bárbara. Asimismo la aplicación de los conocimientos obtenidos favorecerá a la especialización del personal multidisciplinario responsable de las intervenciones preventivas viéndose reflejada en el cambio metodológico del abordaje de los usuarios de los programas de atención. Partiendo que la salud mental es un fenómeno multidimensional y que las teorías fundamentales han sido elaboradas en los países desarrollados, se vuelve prioritario que en nuestro contexto requiera una conceptualización y teorización que se ajuste a nuestra realidad en materia de salud y concretamente en el tema de salud mental.

\section{MÉTODO DE INVESTIGACIÓN}

Diseño de investigación: El tipo de diseño es descriptivo, cuantitativo con estudio de campo, de corte transversal. Este diseño que se selecciono fue para llevar un muestreo polietápico es decir que se escogieron unidad de análisis en tres etapas consecutivas. 
Etapa 1: Se seleccionó los municipios haciendo uso del muestreo no probabilístico es decir que no se rige por aleatoriedad ya que se eligió cada municipio en base a tres criterios fundamentales para la investigación:

1- Densidad poblacional: se ordenó en función al número de pobladores del municipio.

2- Accesibilidad: para el municipio rural, se aseguró que tuvieran acceso en base a carreteras o caminos para poder entrar a realizar el estudio sin muchos problemas y que la distancia no fuera un impedimento para la investigación.

3- Representación geográfica: Consistió en dividir el departamento de Santa Bárbara en cuatro cuadrantes enlistando en cada uno de ellos los municipios que correspondían, para identificar los más representativos del departamento. En esta primera etapa se seleccionó el municipio de Santa Bárbara por ser la cabecera departamental para el área urbana y el municipio Gualala como área rural.

Etapa 2: Consistió en la selección de barrios, colonias, caseríos dentro del municipio urbano, se llevó a cabo mediante un muestreo no probabilísticos que hace uso de los siguientes criterios:

1- Representatividad geográfica: Consistió en dividir el municipio en 4 cuadrantes y enlistando en cada uno de ellos los barrios que corresponden.

2- Densidad poblacional: Se ordenó en función al número depoblación.

3- Procedencia geográfica: Se escogió un barrio o colonia de mayor población del cuadrante.

En esta segunda etapa en base a los criterios en el municipio de Santa Bárbara se escogió; barrios Llano del Conejo, Las Galeras, Gualjoco y El Chaparral.

Etapa 3: Consistió en la selección de casas a ser consultadas; en el área rural se hizo uso de un muestreo probabilístico sistemático para lo cual se hizo uso del factor k que es el número de viviendas entre el número de casos a ser consultado.

Para el área urbana la selección de viviendas fue aleatoria, con un muestreo que se subdividió en cuatro cuadrantes cada barrio de los cuales se extrajo una muestra de viviendas elegidas sistemáticamente mediante el factor $k$. 


\section{Instrumentos}

Los instrumentos previstos para este estudio son: de tamizaje en salud mental (SRQ), cuestionario de caracterización poblacionaly el cuestionario de funcionamiento familiar.

Cuestionario de síntomas para jóvenes y adultos (Escala SRQ): sirve para la detección de alteraciones a la salud mental, diseñado para ser auto-administrado. Es una escala simple y objetiva, fácil de evaluar que cubre aspectos claves de la psicopatología, con preguntas sencillas fáciles de entender. Los ítems se puntúan con cero y uno, indica que el síntoma está presente 0 ausente durante los últimos 30 días.

Encuesta de caracterización poblacional: el propósito de estas encuestas estriba en recopilar información de contexto que permite la realización de análisis detallados y en profundidad de las interrelaciones entre los atributos sociodemográficos tanto a escala de individuos como de los hogares.

Cuestionario del funcionamiento familiar: propuesto por Teresa Ortega, Dolores Cuesta y Caridad Díaz, es una escala tipo Likert que consta de 14 preguntas cada una mide aspectos de cohesión, armonía, comunicación, permeabilidad, afectividad, roles y adaptabilidad que dan como resultado la funcionalidad o disfuncionalidad familiar.

Se estima que le tiempo de llenado del conjunto de instrumentos oscilara entre 17 a 25 minutos, considerando que todos son auto-administrables, no obstante se contó con la asistencia de encuestadores con un nivel educativo universitario quienes previamente se le preparó para dicha actividad.

\section{Población objeto de estudio}

La muestra representativa fue de 300 viviendas del departamento de Santa Bárbara, las cuales se distribuyeron de la siguiente manera; 150 viviendas del área urbana (municipio de Santa Bárbara) y 150 viviendas rurales (municipio de Gualala). Asimismo se distribuyó de manera equitativa entre el género masculino y el género femenino. Los rangos de edad de la población objeto de estudio fueron de 18 años a 30 años, de 31 años a 50 años y de 51 años en adelante. 


\section{Entorno de investigación}

La investigación se llevó a cabo en el departamento de Santa Bárbara ubicada al noroccidente, en específico en la cabecera departamental Santa Bárbara (como área urbana) y municipio de Gualala (como área rural).

\section{Intervenciones}

Los instrumentos de tamizaje empleados para esta investigación fueron validados en dos momentos importantes. En primera instancia se procedió mediante una validación de 60 expertos (psicólogos, psiquiatras y médicos) que realizaron una validación cualitativa, de contenido y de constructo de los instrumentos esto para adaptar los ítems al contexto nacional antes de ir al trabajo de campo. En un segundo momento se procedió a un estudio piloto para validar la adaptación de los ítems a losinstrumentos.

Para el instrumento SRQ se propuso un trabajo de campo que implicó desarrollar un proceso y validación de sensibilidad y especificidad basado en puntos de corte de la curva de ROC. La muestra está compuesta por un total de 352 personas; dividida en dos grupos, el primero compuesto por casos diagnosticados clínicamente con trastornos mentales, siendo necesario 20 sujetos por patología. El segundo grupo estuvo compuesto por 220 sujetos clínicamente sanos definido como grupo controlnormativo.

\section{Análisis estadístico}

En base al principio de representatividad el tamaño de la muestra fue de 300 viviendas, haciendo uso del muestreo por cuotas basados en criterios de inclusión como ser: densidad poblacional, accesibilidad, representación geográfica y que sean personas mayores de 18 años y los criterios de exclusión fueron aplicando el factor $\mathrm{k}$ que es el número de viviendas entre el número de casos a ser consultados.

El tamaño de la muestra ha sido seleccionada con la fórmula de las poblaciones infinitas quedando un total de 300 viviendas; con un intervalo de nivel de confianza del $95 \%$, y un error muestral del $5 \%$.

Las pruebas estadísticas utilizadas para realizar la correlación entre las variables estudiadas fueron la $r$ de Pearson que proporciona una medida numérica de correlación positiva o negativa entre dos variables y la t de student $($ Sig. $\leq 0,05)$ que sirve para establecer diferencias estadísticamente significativa. 
Poblaciones Infinitas $>=100,000$

\begin{tabular}{|l|r|}
\hline Varianza (p) & 75 \\
\hline Varianza (q) & 25 \\
\hline Error muestral (E) & 5.00 \\
\hline Nivel de Confianza (Z) & 2 \\
\hline Tamaño de la Muestra (n) & 300 \\
\hline
\end{tabular}

\section{Consideraciones éticas}

Se hizo uso del consentimiento informado como procedimiento garantizando a los participantes la confidencialidad de los datos y respeto a su identidad, informándoles que los resultados serán utilizados con fines científicos.

\section{RESULTADOS}

Este análisis contiene los principales hallazgos del estudio de prevalencia de trastornos mentales y su relación con la dinámica familiar, el grafico 1 muestra el porcentaje de los trastornos mentales de mayor prevalencia divididos en seis dimensiones que son medidas a través del instrumento de tamizaje de salud mental.

\section{Gráfico 1. Trastornos mentales de mayor prevalencia en la población adulta del departamento de Santa Bárbara, 2016.}

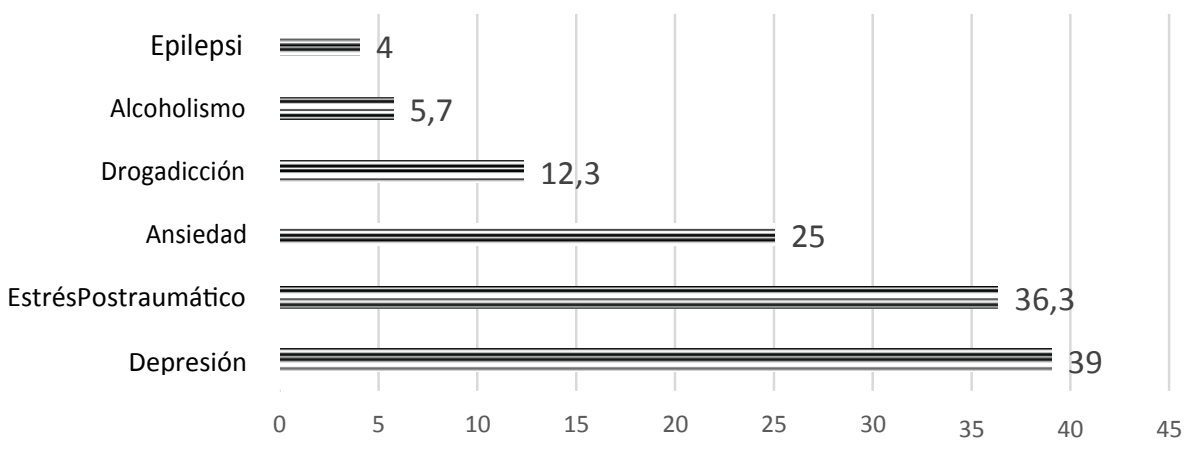

Fuente: Elaboración propia 
Los tres probables trastornos mentales de mayor prevalencia en la población adulta de Santa Bárbara fueron: depresión, estrés postraumático y ansiedad, en menor porcentaje se manifiesta la población que presenta dependencia al alcohol y drogas y también los que manifiestan tener epilepsia. Entre las características sociodemográficas estudiadas con relación a la presencia de sintomatología de probables trastornos mentales se encuentra el género y rango de edad:

Gráfico 2. Prevalencia de probables trastornos mentales según género en la población adulta del departamento de Santa Bárbara.

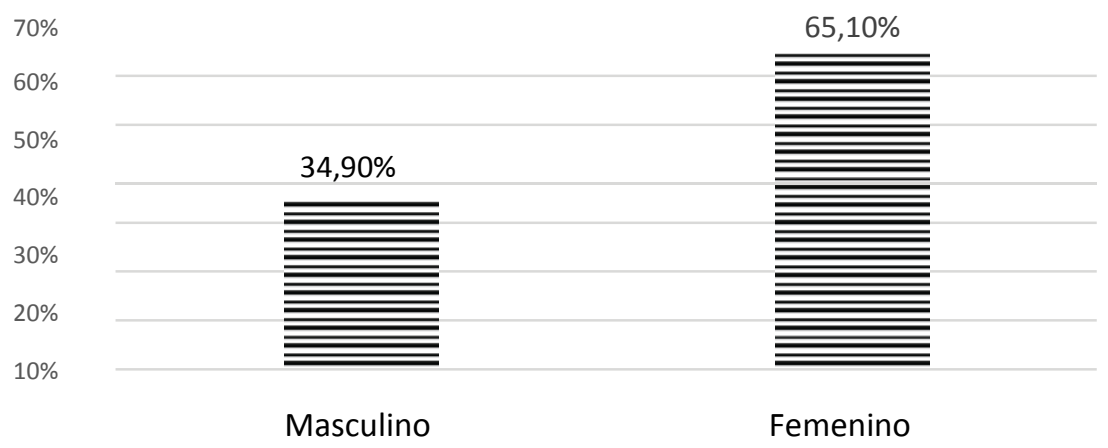

La población femenina (125 mujeres) representa el mayor porcentaje de padecer de un probable trastorno mental en el departamento de Santa Bárbara, así como se han reportado la American Psychological Association (Garcia, 2011) que establece que las mujeres son más propensas a padecer trastornos mentales. Como dato importante se tomaron también rangos de edad para establecer si hay diferencia en las etapas de desarrollo del ciclo vital con la presencia de padecimientos mentales, como lo muestra el gráfico 4.

Gráfico 3. Prevalencia de probables trastornos mentales según rango de edad en la población del departamento de Santa Bárbara

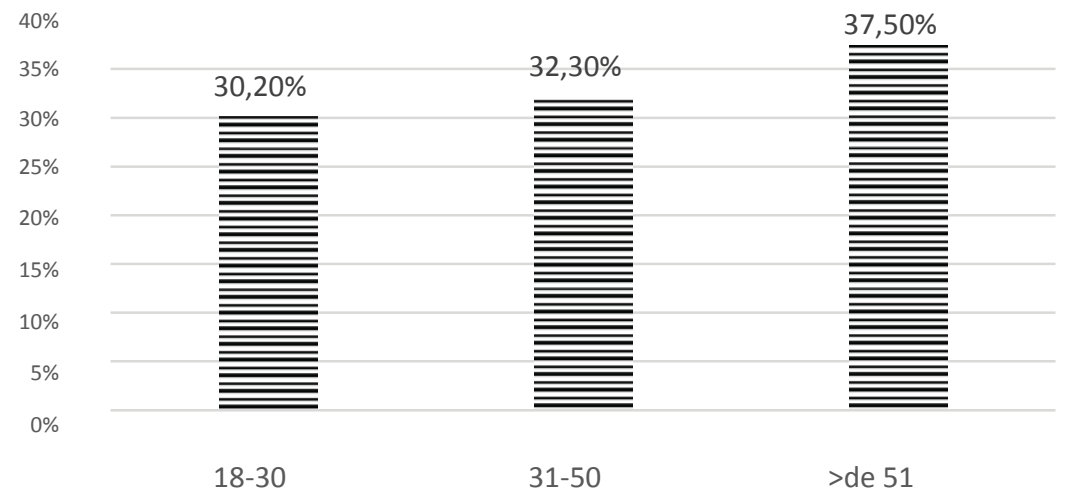


Según el rango de edad hay mayor presencia de sintomatología de probables trastornos mentales en las personas mayores a los 51 años (72 personas), la OMS (2016) estima que la población mundial está envejeciendo rápidamente, entre 2015 y 2050 la proporción de la población mundial mayor de 60 años se multiplicará casi por dos. Por lo tanto como el objetivo principal de esta investigación es establecer la relación entre la dinámica familiar y la presencia de probables trastornos mentales, a continuación se presentan los principales hallazgos, en donde se tomó la media de la población para su respectivo análisis.

\section{Gráfico 4. Dinámica familiar promedio según presencia de trastornos mentales en la población adulta del departamento de Santa Bárbara}

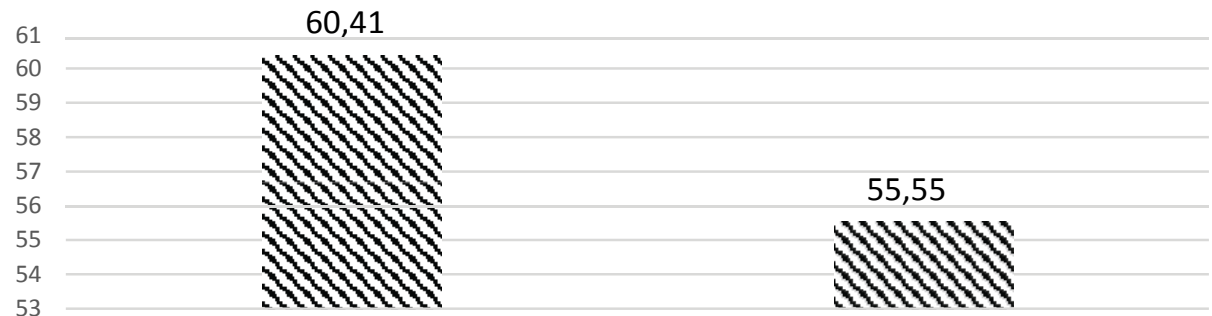

sinpresencia de trastornos mental

PresenciaTrastorno Mental

El gráfico 4 nos pone de manifiesto la media de la población estudiada en Santa Bárbara en donde se observa una diferencia estadísticamente significativa (sig. 0,000 ) entre las familias más funcionales que no presentan probables trastornos mentales y las menos funciones que son más vulnerables a presentar sintomatología de trastornosmentales.

La dinámica familiar se puede categorizar en diferentes niveles de funcionamiento en el núcleo familiar medidos a través de las dimensiones de cohesión, adaptabilidad, permeabilidad, roles, afectividad, armonía y comunicación, a continuación en el gráfico 5 se observa como se muestra la funcionalidad de la familias de la población del departamento de Santa Bárbara.

Las personas del departamento de Santa Bárbara reportan encontrarse en familias funcionales en un alto porcentaje manifestando una dinámica familiar que permite la adaptación de sus miembros, que se encuentran cohesionadas y con una unión familiar física y emocional para el buen funcionamiento de la misma que permite enfrentar diferentes situaciones. 


\section{Gráfico 5. Porcentaje del nivel de funcionamiento de la Dinámica Familiar de la población adulta del departamento de Santa Bárbara.}

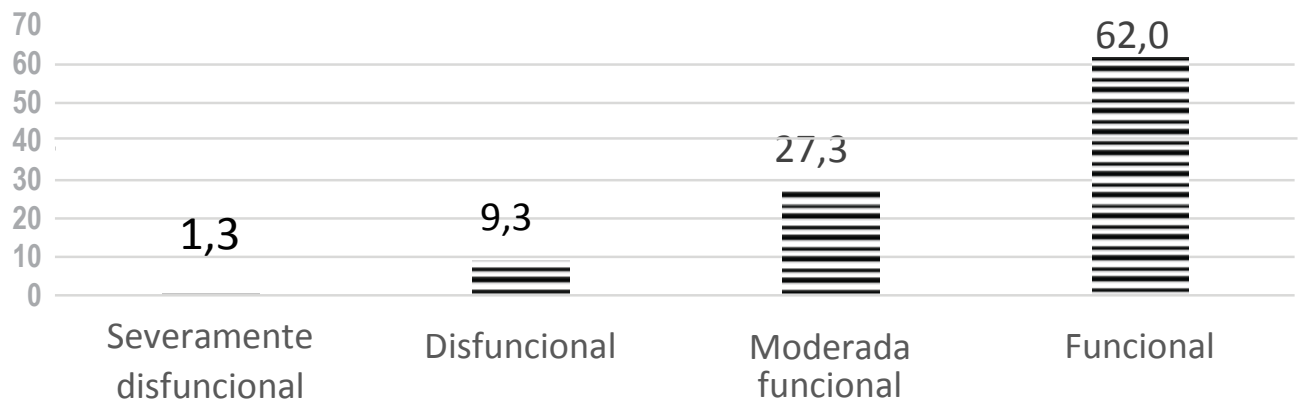

\section{DISCUSIÓN}

Los hallazgos de la investigación nos brindan información y datos relevantes e importantes, se determinó que en un porcentaje alto la población del departamento de Santa Bárbara presenta un probable trastorno mental. Estos resultados son más del doble de lo reportado por la (OMS, 2001) que establece que un $25 \%$ de la población mundial presenta un trastorno mental a lo largo de la vida que puede llegar a incapacitarlo, un estudio de salud mental realizado en Colombia reporta que el $40,1 \%$ de la población ha tenido alguna vez en su vida uno o más de los 23 trastornos basados en el DSM-IV (Posada-Villa, 2004). La prevalencia de trastornos mentales de estas investigaciones nos indica que en el departamento estudiado hay 39 puntos porcentuales por encima de lo que reporta la población a nivel mundial según la OMS. No obstante hay indicios que la prevalencia de los trastornos mentales está aumentando en todo el mundo cerca de un 50\%, pasando de 416 a 615 millones de afectados, casi un $10 \%$ de la población mundial (Organización Médica Colegial de España, 2016).

En el caso de Honduras el estudio realizado por (Chirinos-Flores, 2002) con una muestra representativa y a nivel nacional determino que la prevalencia de trastornos mentales era de un 35\% en la población hondureña este aumento lo atribuyen a las condiciones socioeconómicas, inseguridad ciudadana y desastres naturales. La prevalencia reportada en el 2002 da pauta del porque este aumento en la presencia de sintomatología de probablestrastornosmentaleseneldepartamentodeSantaBárbaraenrelaciónaloestablecidoporlaliteratura mundial. El incremento considerable en la prevalencia de probables trastornos mentales establecida en este estudio sea proba- 
blemente a los cambios que se han presentado en la última década relacionados con el desempleo, destrucción del medio ambiente, alta tasa de violencia e inseguridad (Aranceli, 2006).Asimismo

puede deberse afectada por el contexto, en virtud que la OMS menciona que la salud mental se debe de entender integralmente desde el punto de vista bio-psico-social, en este sentido los trastornos mentales pueden estar siendo exacerbados por factores de tipo social ya que según el observatorio de la violencia de la UNAH ha habido un alza en la tasa de homicidios en Santa Bárbara manifestando que es primera vez que aparece este departamento en el informe con aumento de 149 homicidios de enero a junio del presente año (Denaire, 2016).

Un segundo hallazgo de este estudio reporta que el trastorno mental de mayor prevalencia en la población de Santa Bárbara es la depresión con un 39\%; expertos a nivel mundial consideran la depresión con la enfermedad del siglo XXI ya que sus síntomas van en ascenso y como lo menciona la OMS que se encuentra en los trastornos afectivos de mayor prevalencia (Argensola, 2015). La presencia de sintomatología de estrés postraumático representa un $36,3 \%$ convirtiéndose en el segundo trastorno mental de mayor prevalencia. En Honduras se reporta que 7 de cada 10 hondureños sufren estrés postraumático atribuido al ambiente violento que se presenta en el país, en la actualidad se están presentando muchos casos de personas víctimas de un asalto o extorsión (Martínez, 2014).

Seguidamente se manifiesta la ansiedad con un 25\%, la dependencia a las drogas con $12,3 \%$ y la dependencia al consumo de alcohol $5,7 \%$ y en un menor porcentaje se encuentra la epilepsia (4\%); resultados que coinciden con una serie de estudios de prevalencia de trastornos mentales que establecen que estos son los principales trastornos mentales de mayor prevalencia (Zimmerman, 2000).

En relación a la presencia de probables trastornos mentales según género se refleja más en la población femenina con un $65,1 \%$; como indican numerosos estudios el género femenino tiene mayor riesgo de sufrir una enfermedad mental que los hombres ya que se relaciona con factores que predisponen al desarrollo de una patología como el papel que juegan las hormonas en la mujer más en la edad reproductiva, factores sociales como el hecho de sobrecarga laboral y tareas domésticas así como o vivir una situación de violencia intrafamiliar, es decir que las mujeres presentan un mayor número de acontecimientos estresantes (Rodriguez, 2013).

En cuanto al rango de edad hay mayor presencia de sintomatología de probables 
trastornos mentales las personas mayores a los 51 años con un 37,5\%. Según estima la OMS (2016) la población mundial está envejeciendo rápidamente, entre 2015 y 2050 la proporción de la población mundial mayor de 60 años se multiplicara pasando del $12 \%$ al $22 \%$ y aproximadamente un $15 \%$ de los adultos de 60 años 0 mayores sufren algún trastorno mental.

Por otro lado la dinámica familiar es un factor determinante en la conservación de la salud mental o en la aparición de la enfermedad entre sus miembros (Louro, 2005). En esta investigación se encontró que la mayoría de las familias en el departamento de Santa Bárbara son funcionales en un $62 \%$ pero alrededor del $19.3 \%$ indican pertenecer a familias disfuncionales. En el análisis de resultados con la prueba $t$ de student, considerando como dato importante la media de la población se observa que hay diferencias estadísticamente significativas $($ Sig. 0,000$)$ entre la variable de la dinámica familiar y los probables trastornos mentales encontrando que en el grupo de personas con mayor funcionalidad familiar no presentan probables trastornos mentales (60.41) y la población con menor funcionalidad familiar si presenta probables trastornos mentales (55.55). Es decir que el funcionamiento familiar saludable implica el desarrollo de una dinámica adecuada en la familia, si el sistema familiar cumple plenamente la seguridad económica, afectiva, social, espiritual y de modelos sexuales genera nuevos individuos en la sociedad en una homeostasis sin tensión mediante una comunicación apropiada y basada en el respeto de las relaciones intrafamiliares (Ávalos-Garcìa,2013).

La dinámica familiar de la población adulta del departamento de Santa Bárbara está basada en las dimensiones medidas por la escala FF-SIL en las cuales la cohesión, afectividad, comunicación y adaptabilidad son estadísticamente significativas y mantienen una correlación negativa sin embargo no son factores causales de la presencia de sintomatología de probables trastornos mentales sino que actúan como un factor de protección que ayuda a mantener un buen nivel de funcionamiento de las personas en las familias y en la sociedad.

En conclusión, los hallazgos de este estudio demuestran un alza de sintomatología de probables trastornos mentales en la población del departamento de Santa, esto tiene implicaciones para los servicios de atención a la población adulta por lo que se debería contar en los centros de salud con personal capacitado en atención a la salud mental, este personal deberá estar preparado para detectar y tratar este tipo de problemas y poder así mismo hacer la referencia apropiada a los servicios de atención especializados en la capital del país para la atención oportuna y su respectivo tratamiento. 
A la vez asegurar la apertura de espacios en los centros de salud, cesares y cesamos para que se cuente con la asistencia de un psicólogo como también en los centros educativos, como parte de una prevención y promoción primaria que contribuya al fomento de los hábitos protectores y a la reducción de los comportamientos de riesgo mejorando así la calidad de vida de la población en general (Alarcón, 2007).

Aun cuando la salud forma parte del plan de nación de país 2021 donde uno de los objetivos es reducir las inequidades y mejorar la calidad de vida de la población de Honduras (Secretaría de Salud, 2005), así como también la salud pública es vista como la encargada de proteger y mejorar la salud de las personas, es preocupante que la prevalencia de estos trastornos sea tan elevada y al mismo tiempo tan invisible ya que en nuestro país no se cuenta con una red de atención en salud mental por el escaso presupuesto que hay para este,yporloqueconciernelaausenciadeaccesibilidadaestosserviciosenlosmunicipiosdeSantaBárbara, sumando a esto el programa de salud mental desaparece en el nuevo modelo de servicio en salud, es decir que no existe un sistema de prevención y promoción en salud mental a nivel primario. Finalmente es indispensable la reorganización y elaboración de nuevas propuestas en salud mental y programas de intervención psico-social que puedan adoptar políticas eficaces para la toma de decisiones que garanticen una atención en salud que sea integral, descentralizada, participativa y preventiva a favor de las comunidades especialmente a las más vulnerables por el sistema, ya que en la medida que se logre identificar de manera temprana los factores de riesgo de presentar trastornos mentales permitirá evitar situaciones de crisis y, esto ayudara a mejorar y hacer efectivo el derecho a una calidad de una vida digna.

\section{CONCLUSIONES}

1. Mediante el instrumento $S R Q$, se determinó que la prevalencia de probables trastornos mentales en la población adulta del departamento de Santa Bárbara es de un $39 \%$ la depresión, seguido del estrés postraumático $(36,3 \%)$, ansiedad (25\%), dependencia a las drogas (12,3\%), y al consumo de alcohol $(5,7 \%)$ y finalmente la epilepsia (4\%).

2. La caracterización de las personas con probables trastornos mentales de Santa Bárbara corresponde en su mayoría a las mujeres y a la población en un rango de edad mayor a los 51 años, asimismo la presencia de sintomatología de trastornos mentales se enfatiza más en el casco urbano sin embargo se refleja un $0.5 \%$ de diferencia con el árearural. 
3. No se encontró una relación causal directa en la presencia de los probables trastornos mentales y la dinámica familiar, sin embargo se obtuvo una correlación negativa $(-0,033)$ y una diferencia estadísticamente significativa $($ sig.0,000) entre las familias más funcional es sin presencia de trastornos mentales con un promedio en su dinámica familiar de 60.41y las familias con menor funcionalidad con presencia de trastornos mentales con un promedio de55.55.

4. La funcionalidad en relación a la dinámica familiar de la población de Santa Bárbara, manifiestan estar en un $62 \%$ en familias funcionales, seguidamente de un $27.3 \%$ en un nivel de moderadamente funcional y con un menor porcentaje se encuentra el $9 \%$ en familias disfuncionales y $1.3 \%$ severamente disfuncionales.

5. En relación al promedio de la dinámica familiar según la presencia de probables trastornos mentales más frecuentes hay diferencias estadísticamente significativas a un nivel de confianza de $95 \%$, reportando que en la población con mayor funcionalidad no hay sintomatología relacionada con ansiedad, depresión, dependencia al alcohol y drogas y estrés postraumático.

6. Entre las dimensiones de la dinámica familiar medidas a través de la escala de funcionamiento familiar FF-SIL, que muestran una correlación negativa y considerando el 95\% de confianza, en la presencia de trastornos mentales fueron cohesión, comunicación, afectividad, adaptabilidad, roles y armonía en la presencia de ansiedad, depresión, dependencia al alcohol y drogas y estrés postraumático.

\section{AGRADECIMIENTOS}

Agradezco a la Maestría en Psicología Clínica y a la Dirección de Investigación Científica de la UNAH por su apoyo en la realización de este proyecto.

\section{REFERENCIAS BIBLIOGRÁFICAS}

Alarcón, F. (2007). Psicología social de la salud: Promoción y prevención. Revista Latinoamericana de Psicología, 40(1), 174-175.

Aranceli, S. P. (2006). Evaluación de niveles, situaciones generadoras y manifestaciones de estrés académico de tercer y cuarto año de la Facultad de Estomatología. Estomatológica Herediana, 16(1), 15-20.

Argensola. (Junio de 2015). Argensola, Psicología y Psicoterapia. Obtenido de La Depresión, ¿La Enfermedad del Siglo XXI?: http://psicologiaypsicoterapia.com/la-depresion-la-epi- 
demia-del-siglo-xxi/

Ávalos, M. (2013). Síndrome de Burnout, funcionalidad familiar y factores relacionados en personal médico y de enfermería de un hospital de Villahermosa, Tabasco, México. Revista Científica de América Latina y el Caribe, 12(2),48-49.

Chirinos, A., Munguia, A., Lagos, A., Salgado, J., Reyes, A., Padgett, D., \& Donaire, L. (2002). Prevalencia de Trastornos Mentales en la población mayor de 18 años en 29 comunidades urbanas de Honduras, 2001 Tegucigalpa, Honduras. Revista Médica Postgrado UNAH, 7(1), 42-48.

Denaire, L . (04 de Octubre de 2016). Córtes y Francisco Morazán los departamentos más violentos. Diario la Prensa. Recuperado de http://www.laprensa.hn/honduras/ 1005594-410/cortés-registra-la-tasa-de-homicidios-más-alta-según-observatorio-de

García, M. (7 de Septiembre de 2011). Hombres y Mujeres tienden a sufrir enfermedades mentales distintas (Mensaje en un blog) . Recuperado de http://www.tendencias21.net/Hombres-y-mujeres-tienden-a-sufrir-enfermedades-mentales-distintas_a7384.html Louro, I. (2005). Modelo de Salud del Grupo Familiar. Revista Cubana Pública, 31(4), 129. Martínez, J. (13 de Julio de 2014). Deprimidos hondureños por falta de "pisto". Diario La Tribuna. Recuperado de http://www.latribuna.hn/2014/07/13/deprimidos-hondurenos-por-falta-de-pisto/

Organización Médica Colegial de España. (13 de Abril de 2016). Medicosypacientes.com. Obtenido de http://www.medicosypacientes.com/articulo/los-trastornos-mentales-afectan-al-10-por-ciento-de- la-poblacion-mundial

Posada-Villa, J., Aguilar-Gaxiola, S., Magaña, C., \& Gomez, L. (2004). Prevalencia de Trastornos Mentales y Uso de servicios. Resultados preliminares del Estado Nacional de Salud Mental. Colombia,2003. Revista Colombiana de Psiquiátria, 33(3): 241-262.

Rodríguez, J. (23 de Mayo de 2013). Eroski Consumer. Obtenido de La salud mental de la mujer: http://www.consumer.es/web/es/salud/prevencion/2013/05/26/216742.php

Secretaría de Salud. (2005). Plan nacional de salud 2021. Recuperado de http://www.bvs.hn/Honduras/pdf/Politicas_Nacionales_Salud-Honduras_Plan_Nacional_2021.pdf

Zimmerman, M., McDermut, W., \& Mattia, J. (2000). Frequency of anxiety disorders in psychiatric outpatients with major depressive disorder. American Journal Of Psychiatry, 157(8), 1377-1340. 